\title{
BMJ Open Non-adherence to immunosuppressants following renal transplantation: a protocol for a systematic review
}

\author{
Abigail Hucker, ${ }^{1}$ Frances Bunn, ${ }^{2}$ Lewis Carpenter, ${ }^{1}$ Christopher Lawrence, ${ }^{3}$ \\ Ken Farrington, ${ }^{3}$ Shivani Sharma ${ }^{1}$
}

To cite: Hucker A,

Bunn F, Carpenter L, et al. Non-adherence to immunosuppressants following renal transplantation: a protocol for a systematic review. BMJ Open 2017;7:e015411. doi:10.1136/ bmjopen-2016-015411

- Prepublication history for this paper is available online. To view these files please visit the journal online (http://dx.doi. org/10.1136/bmjopen-2016015411).

Received 14 February 2017

Revised 9 May 2017

Accepted 2 June 2017

\section{CrossMark}

${ }^{1}$ Department of Psychology and Sport Sciences, School of Life and Medical Sciences, University of Hertfordshire, Hatfield, UK ${ }^{2}$ Centre for Research in Primary and Community Care, University of Hertfordshire, Hatfield, UK

${ }^{3}$ Renal Unit, Lister Hospital, Stevenage, Hertfordshire, UK

Correspondence to

Dr Shivani Sharma;

s.3.sharma@herts.ac.uk

\section{ABSTRACT}

Introduction Adherence to immunosuppressant medication is essential for renal transplant recipients. This review aims to summarise what is known about non-adherence, with a view to providing comprehensive evidence to inform strategies aimed at advancing adherent behaviour.

Methods and analysis A systematic review of quantitative studies that report adherence to immunosuppressants in adult (over 18 years) renal transplant recipients. The review will follow the PRISMA (Preferred Reporting Items for Systematic Reviews and Meta-analyses) guidelines; study quality will be assessed using the Downs and Black checklist. Systematic searches will be completed across relevant databases. Two reviewers will independently extract data using a predefined data extraction form. We will summarise the operationalisation of adherence across studies and use narrative synthesis to identify factors associated with nonadherence. A meta-analysis will be conducted if there is sufficient homogeneity, and available data, across studies to estimate the prevalence of non-adherence in renal transplant recipients. Heterogeneity will be assessed using the $I^{2}$ test. Survival analysis will be conducted to estimate hazard ratios to explore the impact of non-adherence on graft survival, graft failure and patient survival.

Ethics and dissemination Findings will be published in a peer-reviewed journal and disseminated at conferences for professionals and researchers. Review outcomes will help support clinical practice by highlighting the extent of non-adherence among adults, and in doing so, signpost the need for suitable intervention.

Trial registration number PROSPERO registration number (CRD42016038751).

\section{INTRODUCTION}

Chronic kidney disease (CKD) is a longterm condition characterised by progressive decline in kidney function over time ${ }^{1}$ and often occurs in association with other illnesses. $^{2}$ End-stage renal disease (ESRD) occurs within stage five CKD, at which point patients require renal replacement therapy (RRT) in order to survive. For the majority of patients, kidney transplantation is the best and most cost-effective option. ${ }^{3}$ At the end

\section{Strengths and limitations of this study}

- Adherence to immunosuppressant medication is essential for renal transplant recipients; however, there is a need for an up-to-date synthesis of current evidence on non-adherence in this group.

- Findings from this review could help inform patient information needs and the design and delivery of psychosocial support to improve adherence for renal transplant recipients.

- This review will only include studies conducted in English due to the complexity involved in translation of papers.

of 2012, 55000 patients were receiving RRT in the UK, with $50 \%$ having a functioning kidney transplant. ${ }^{4}$

Renal transplant patients must take immunosuppressant medication to prevent rejection of the organ. ${ }^{5}$ A combination of two or three different immunosuppressants are taken on a long-term basis to prevent rejection; however, as with all medications, patients can experience side effects. ${ }^{6}$ This can include an increased risk of infection, diabetes, increased susceptibility to certain types of cancers, increased blood pressure and weight gain. ${ }^{7}$ It is also true that the process of immunosuppression itself may add to the burden of comorbidity. ${ }^{8}$ Despite the nuances of medication, adherence to the immunosuppressive regimen is vital to provide the kidney with the best chance of survival and function following transplantation.

Adherence in this context refers to 'the extent to which the patient's behaviour matches agreed recommendations from the prescriber'. ${ }^{9}$ Adherence can be measured in a number of different ways, including self-report, observation of medication intake, reports from clinicians and electronic monitoring. ${ }^{10}$ Electronic monitoring is regarded as the most accurate option for measuring adherence in research as it is both a sensitive 
and objective measure. ${ }^{10}$ However, self-reported non-adherence has been shown to be strongly predictive of adherence measured by electronic monitoring, and therefore useful for identifying patients at greater risk for non-adherence in clinical practice. ${ }^{11}$

Non-adherence to immunosuppressive medication is a major risk factor for poor clinical outcomes post-transplantation; however, it remains a common issue in this patient population, identified as the second most common cause of late graft failure in renal transplant patients. ${ }^{12}$ A review based on 38 articles ${ }^{13}$ estimated that the rate of non-adherence lies between $28 \%$ and $52 \%$. In a meta-analysis, the odds of graft failure were seven times greater in non-adherent patients compared with adherent patients. ${ }^{14}$ Factors identified as determinants of non-adherence post-transplant ${ }^{14}$ were consistent with more recent literature, such as younger age, being unmarried and perceiving low social support, ${ }^{11}{ }^{13}$ high emotional distress and high transplant-related stress. ${ }^{15}$ In addition to this, having a donor graft from a living relative has also been identified as a risk factor for non-adherence. ${ }^{11}$ Furthermore, ill health resulting from non-adherence is costly for the National Health Service (NHS), since dialysis services are considerably more expensive than those required to support a functioning transplant. ${ }^{15}$ Moreover, a prerequisite for optimal adherence is access to treatment; however, previous literature has highlighted that cost-related non-adherence is an issue among renal transplant recipients in localities such as the USA, as patients do not benefit from insurance coverage to support access to immunosuppressive medication, and are therefore at greater risk of graft failure. ${ }^{16}$

There are a number of reasons why patients may not adhere to their immunosuppressant regimen. In addition to those mentioned above, unintentional and intentional non-adherence have been identified as issues in this patient population. Unintentional non-adherence is significantly more common than intentional non-adherence, ${ }^{17}$ and most commonly occurs when patients find themselves outside of their normal daily routine. In contrast, intentional non-adherence occurs when patients have concerns about their medication regimen. Psychological distress, notably anxiety, hostility and depression have been identified as having an impact on medication adherence, ${ }^{18}$ with depression also being associated with higher intentional non-adherence. ${ }^{17}$ In addition, non-adherent patients have a greater symptom burden, higher distress levels ${ }^{19}$ and report higher frequency of stress. ${ }^{20}$ Hence, renal transplant recipients may require a range of supports to help them maintain a lifestyle associated with their health status. A recent systematic review evaluated interventions to improve medication adherence in adult renal transplant patients, ${ }^{21}$ suggesting that those targeting behavioural risk factors or multidimensional interventions $^{22} 23$ combining educational, behavioural and emotional factors are more likely to be effective in improving non-adherence to medication.
Clearly non-adherence to immunosuppressive medication is common and a major risk factor for poor outcomes post-transplantation. The consequences of non-adherence are far reaching for patients in terms of reduced health-related quality of life and survival. Added to this they entail a significant cost burden-related to increased care needs. ${ }^{16}$ There are two core reviews available, published in $2003^{15}$ and $2004^{14}$ which aim to highlight the extent of non-adherence in this patient group. It is fair to say, though that progressive recognition of non-adherence in renal care settings has resulted in a larger evidence base. A synthesis of the existing body of research in the area would therefore be welcome to guide clinical practice and to further inform the nature of intervention.

In the light of this, the current paper details proposals for a systematic review and meta-analysis that will provide the most comprehensive evidence synthesis of what is known about non-adherence in adult renal transplant recipients. In doing so, the review will:

- Summarise the methods used to assess non-adherence and discuss how non-adherence is operationalised across studies.

- Identify the prevalence of non-adherence by pooling data from across studies and considering whether reported non-adherence varies as a function of measurement source.

- Provide a narrative summary of the main factors associated with non-adherence.

- Estimate the impact of psychological correlates such as depression or anxiety and illness perceptions on non-adherence.

- Estimate the impact of non-adherence on graft failure, graft survival and mortality.

\section{METHODS AND DESIGN}

This protocol has been written using the PRISMA-P (Preferred Reporting Items for Systematic Reviews and Meta-analyses Protocols) guidelines and has been registered on the PROSPERO database (registration number: CRD42016038751).

\section{Eligibility criteria}

This review will only include English language literature. Study inclusion and exclusion criteria are given below.

\section{Types of studies}

This review will include published peer-reviewed studies and grey literature. All quantitative studies that examine non-adherence to immunosuppressant's in renal transplant patients will be included (cross-sectional, cohort and case series studies will be included). Randomised controlled trials will not be included, as this systematic review is not analysing interventions. All studies must include a measure of non-adherence.

\section{Participants}

This review will include patients aged 18 and over who have received a solid organ kidney transplant. Participants will be included irrespective of graft function (functioning or 
failed) at the time of entry into the study. Children and adolescents will be excluded from this review as evidence suggests that different challenges contribute to non-adherence in this group. ${ }^{24}$

\section{Outcomes}

\section{Primary outcomes}

Studies will be included if one of the outcomes is the degree of non-adherence to immunosuppressants. It is likely that this will be measured in a number of ways including self-report, electronic monitoring of pill bottles and observations.

\section{Secondary outcomes}

For the studies that meet the primary inclusion criteria, the following outcomes will also be assessed if sufficient information is available: whether non-adherence is intentional or unintentional (forgetting), risk factors for non-adherence as reported in the studies, for example, age, time since transplantation, comorbidities, psychological correlates of non-adherence, for example, depression, illness perceptions, and clinical outcomes such as graft failure, graft survival and mortality.

\section{Information sources}

Searches will include the following electronic databases: PubMed, Scopus, CINAHL, The Cochrane library, PsychARTICLES and Google scholar. There will be no date limits on the searches in all. In addition, lateral search techniques will be used, such as checking reference lists for related articles and using Google scholar to conduct key word and citation searches. Furthermore, contact with experts in the field will identify grey literature and ongoing studies.

\section{Search strategy}

A combination of free text terms and medical subject heading $(\mathrm{MeSH})$ search terms will be used in all databases. The search strategy to be used in PubMed is included in table 1 . This will be adapted accordingly to the subject headings of the remaining databases.

\section{Study screening and selection process}

Citations from all database searches will be imported into Endnote reference management software. A check will be run to identify, note and remove any duplicate citations.

Retrieved records will be screened by title and abstract by two reviewers independently in order to assess suitability for inclusion. Any disagreements will be resolved by discussion or consultation with a third reviewer. Full texts of potentially relevant citations will be independently assessed for inclusion in the review using a piloted screening proforma. A second reviewer will independently screen $25 \%$ of the titles and abstracts and full texts for quality assurance. If any relevant information on eligibility is missing, study authors will be contacted. Reasons for excluding studies will be recorded.

\begin{tabular}{|c|c|c|}
\hline Concept & No & Search terms \\
\hline \multirow{4}{*}{$\begin{array}{l}\text { Concept } \\
\text { 1: Population }\end{array}$} & 1 & kidney transplantation (MeSH) \\
\hline & 2 & $\begin{array}{l}\text { "kidney transplant" (free-text } \\
\text { term) }\end{array}$ \\
\hline & 3 & "renal transplant*"(free-text term) \\
\hline & 4 & OR $[1-3]$ \\
\hline \multirow{12}{*}{$\begin{array}{l}\text { Concept 2: } \\
\text { Adherence (outcome) }\end{array}$} & 5 & adherence, medication (MeSH) \\
\hline & 6 & adherence (free-text term) \\
\hline & 7 & non-adherence (free-text term) \\
\hline & 8 & noncompliance (free-text term) \\
\hline & 9 & compliance (free-text term) \\
\hline & 10 & $\begin{array}{l}\text { "treatment refusal" (free-text } \\
\text { term) }\end{array}$ \\
\hline & 11 & $\begin{array}{l}\text { "patient compliance” (free-text } \\
\text { term) }\end{array}$ \\
\hline & 12 & $\begin{array}{l}\text { "medical compliance" (free-text } \\
\text { term) }\end{array}$ \\
\hline & 13 & $\begin{array}{l}\text { "medical adherence" (free-text } \\
\text { term) }\end{array}$ \\
\hline & 14 & $\begin{array}{l}\text { "medical non-adherence" (free- } \\
\text { text term) }\end{array}$ \\
\hline & 15 & concordan* (free-text term) \\
\hline & 16 & OR [5-15] \\
\hline \multirow{3}{*}{$\begin{array}{l}\text { Concept 3: } \\
\text { Immunosuppression }\end{array}$} & 17 & immunosuppression (MeSH) \\
\hline & 18 & $\begin{array}{l}\text { immunosuppress* (free-text } \\
\text { term) }\end{array}$ \\
\hline & 19 & OR $[17-18]$ \\
\hline $\begin{array}{l}\text { All concepts } \\
\text { combined }\end{array}$ & 20 & 4 AND 16 AND 19 \\
\hline
\end{tabular}

\section{Data collection process}

A data extraction form will be used to extract the relevant information from each study to be included in the review. The form will be created in Microsoft Excel. Two reviewers will independently extract the relevant information from the full text papers into the data extraction table. This will be pilot tested on a few studies prior to the review to ensure consistency between the two reviewers and to ensure the correct information has been included. The use of two reviewers for data extraction will be used to reduce the risk of error. Any disagreement will be resolved by discussion with a third reviewer.

\section{Data items}

Data will be extracted using the following categories:

- General information: date of data extraction, study title, author and year of publication, country of origin

- Study methods/characteristics: study design (including how adherence was defined and measured), study aims and research questions, inclusion/ exclusion criteria, sampling methods 
- Demographics about the participants (age, sex, ethnicity, socio-economic characteristics)

- Number of non-adherent patients

- Number of transplants

- Outcomes of the study, correlates and any other clinical measures (such as graft survival, number of graft failures (assessed as caused by non-adherence) and mortality/survival)

\section{Outcomes and prioritisation}

The primary outcome will be the degree of non-adherence to immunosuppressants. For studies that meet the primary inclusion criteria, the following outcomes will also be assessed if available: whether non-adherence is intentional or unintentional (eg, forgetting), which is likely to be reported via self-report; psychological correlates such as depression or anxiety (measured using any validated scale); illness perceptions; clinical outcomes (eg, graft rejection, graft failure and mortality).

\section{Risk of bias in individual studies}

Studies will be rated for quality assurance using the Downs and Black ${ }^{25}$ checklist for non-randomised studies, casecontrol, cross-sectional and cohort studies. This consists of five domains: study quality, external validity, study bias, confounding and selection bias and study power. One reviewer $(\mathrm{AH})$ will rate all the studies, and a second reviewer will independently rate $25 \%$ of the studies chosen at random. Any discrepancies between reviewers will be resolved by discussion with a third reviewer.

\section{Data synthesis}

A narrative synthesis will be used to summarise the different methods of measuring adherence. In addition, we will investigate how studies define non-adherence, and whether this is consistent across studies. A meta-analysis will also be included in this review, if there is sufficient homogeneity across outcomes and available data. Where appropriate, we will use meta-analysis to pool together study outcomes to assess: (1) the prevalence of non-adherence; (2) factors associated with non-adherence (such as illness perceptions and depression); (3) impact of non-adherence on graft survival, graft failure and patient survival. Heterogeneity will be assessed using the $\mathrm{I}^{2}$ test. ${ }^{26}$ An $\mathrm{I}^{2}$ value of $25 \%$ is categorised as low, 50\% moderate and $75 \%$ high. ${ }^{27}$ In addition, stratified analysis and/or metaregression will be conducted to compare measurement methods, dependent on the number of categories and number of studies within each category. This analysis will be conducted using Stata (version 13). As this review will include observational studies, we anticipate using random effects meta-analysis. It is likely that studies will vary due to differences in patient populations, and while provisions have been made to account for this variability, it is unlikely that this will be controlled for its entirety through the use of metaregression or subgroup analyses.

\section{Metabias}

Stratified analysis or metaregression will be used to compare studies that scored high versus those that scored low on the Downs and Black quality assessment tool. Publication bias will be investigated through the use of funnel plots.

\section{Confidence in cumulative estimate}

The quality of evidence for outcomes will be assessed using the "Grading of Recommendations Assessment, Development and Evaluation' (GRADE) approach. ${ }^{28}$ Quality will be determined as high, moderate, low or very low. ${ }^{29}$

\section{DISCUSSION AND DISSEMINATION}

Non-adherence to immunosuppressants is a major risk factor for poor outcomes in renal transplant recipients and so has gained increasingly more research attention as an issue that needs tackling. The findings from this systematic review will be disseminated via a variety of routes including publication in a peer-reviewed journal, conference presentations and through the clinical and academic networks of the team. The findings will be used to inform researchers and medical professionals about the extent of non-adherence in renal transplant recipients, alongside whether estimates vary depending on how information on adherence is sourced. The comprehensive evidence synthesis will also summarise the state of knowledge in relation to factors associated with non-adherence and so by bringing together a larger evidence base, may be able to better inform strategies aimed at enhancing patient's self-care behaviour. Information gleaned from this review could, for example, help inform patient information needs and design and delivery of psychosocial support to improve adherence, by providing an indication of what areas should be targeted to address this issue and when.

Contributors SS conceived the study concept. SS, FB and LC provided expertise in systematic review methodology and meta-analysis. $\mathrm{CL}$ and $\mathrm{KF}$ helped ensure that the aims and scope of the review are clinically relevant. AH drafted the systematic review protocol manuscript. All authors provided feedback during the development of the protocol and approved the final manuscript.

Competing interests None declared.

Provenance and peer review Not commissioned; externally peer reviewed.

Data sharing statement This systematic review protocol has been registered on the PROSPER0 database (registration number: CRD42016038751). This protocol has been written using the PRISMA-P guidelines. Once completed, the final review and data will be submitted for publication.

Open Access This is an Open Access article distributed in accordance with the Creative Commons Attribution Non Commercial (CC BY-NC 4.0) license, which permits others to distribute, remix, adapt, build upon this work non-commercially, and license their derivative works on different terms, provided the original work is properly cited and the use is non-commercial. See: http://creativecommons.org/ licenses/by-nc/4.0/

(c) Article author(s) (or their employer(s) unless otherwise stated in the text of the article) 2017. All rights reserved. No commercial use is permitted unless otherwise expressly granted. 


\section{REFERENCES}

1. National Kidney Foundation. About chronic kidney disease. 2014 https://www.kidney.org/kidneydisease/aboutckd (accessed Mar 2016).

2. Sarnak MJ, Levey AS. Cardiovascular disease and chronic renal disease: a new paradigm. Am J Kidney Dis 2000;35:S117-31.

3. Abecassis M, Bartlett ST, Collins AJ, et al. Kidney transplantation as primary therapy for end-stage renal disease: a National Kidney Foundation/Kidney Disease Outcomes Quality Initiative (NKF/ KDOQITM) conference. Clin J Am Soc Nephrol 2008;3:471-80.

4. National Kidney Federation. Facts \& figures for kidney patients. 2014 http://www.britishrenal.org/BritishRenalSociety/files/26/26a2c68fed7c-4e57-bc01-e97526a86c04.pdf (accessed Mar 2016).

5. National Kidney Federation. What is a kidney transplant? - What drugs are needed? 2000 http://www.kidney.org.uk/organ-donation/ medical-info-transplant-txwhat/medical-info-transplant-txdrugs/ (accessed Mar 2016).

6. National Kidney Foundation. Immunosuppressants. 2015 https:// www.kidney.org/atoz/content/immuno (accessed Feb 2016).

7. The National Kidney Foundation. Care after kidney transplant. 2016 https://www.kidney.org/atoz/content/immunosuppression (accessed Oct 2016).

8. Silkensen JR. Long-term complications in renal transplantation. J Am Soc Nephrol 2000;11:582-8.

9. Horne R, Weinman J, Barber N. 2005. Concordance, adherence and compliance in medicine taking. London: NCCSDO: 40-46.

10. Schäfer-Keller P, Steiger J, Bock A, et al. Diagnostic accuracy of measurement methods to assess non-adherence to immunosuppressive drugs in kidney transplant recipients. Am J Transplant 2008;8:616-26.

11. Denhaerynck K, Steiger J, Bock A, et al. Prevalence and risk factors of non-adherence with immunosuppressive medication in kidney transplant patients. Am J Transplant 2007;7:108-16.

12. Loghman-Adham M. Medication noncompliance in patients with chronic disease: issues in Dialysis and renal transplantation. $A m \mathrm{~J}$ Manag Care 2003;9:155-73.

13. Denhaerynck K, Dobbels F, Cleemput I, et al. Prevalence, consequences, and determinants of nonadherence in adult renal transplant patients: a literature review. Transpl Int 2005;18:1121-33.

14. Butler JA, Roderick P, Mullee M, et al. Frequency and impact of nonadherence to immunosuppressants after renal transplantation: a systematic review. Transplantation 2004;77:769-76.

15. Jindal RM, Joseph JT, Morris MC, et al. Noncompliance after kidney transplantation: a systematic review. Transplant Proc 2003;35:2868-72.
16. Gill JS, Tonelli M. Penny wise, pound foolish? coverage limits on immunosuppression after kidney transplantation. N Engl J Med 2012;366:586-9.

17. Griva K, Davenport A, Harrison M, et al. Non-adherence to immunosuppressive medications in kidney transplantation: intent vs. forgetfulness and clinical markers of medication intake. Ann Behav Med 2012;44:85-93.

18. Achille MA, Ouellette A, Fournier S, et al. Impact of stress, distress and feelings of indebtedness on adherence to immunosuppressants following kidney transplantation. Clin Transplant 2006;20:301-6.

19. Lee SY, Chu SH, Oh EG, et al. Low adherence to immunosuppressants is associated with symptom experience among kidney transplant recipients. Transplant Proc 2015;47:2707-11.

20. Brito DC, Marsicano EO, Grincenkov FR, et al. Stress, coping and adherence to immunosuppressive medications in kidney transplantation: a comparative study. Sao Paulo Med J 2016;134 http://www.scielo.br/scielo.php?pid=S1516-31802015005008106\& script=sci_arttext.

21. Low JK, Williams A, Manias E, et al. Interventions to improve medication adherence in adult kidney transplant recipients: a systematic review. Nephrol Dial Transplant 2015;30:1-10.

22. Chisholm MA, Mulloy LL, Jagadeesan M, et al. Impact of clinical pharmacy services on renal transplant patients' compliance with immunosuppressive medications. Clin Transplant 2001;15:330-6.

23. Chisholm-Burns MA, Spivey CA, Graff Zivin J, et al. Improving outcomes of renal transplant recipients with behavioral adherence contracts: a randomized controlled trial. Am J Transplant 2013;13:2364-73.

24. Dobbels F, Ruppar T, De Geest S, et al. Adherence to the immunosuppressive regimen in pediatric kidney transplant recipients: a systematic review. Pediatr Transplant 2010;14:603-13.

25. Downs $\mathrm{SH}$, Black $\mathrm{N}$. The feasibility of creating a checklist for the assessment of the methodological quality both of randomised and non-randomised studies of health care interventions. J Epidemiol Community Health 1998;52:377-84.

26. Higgins JP, Thompson SG. Quantifying heterogeneity in a metaanalysis. Stat Med 2002;21:1539-58.

27. Higgins JP, Thompson SG, Deeks JJ, et al. Measuring inconsistency in meta-analyses. BMJ 2003;327:557-60.

28. Guyatt G, Oxman AD, Akl EA, et al. GRADE guidelines: 1. introduction-GRADE evidence profiles and summary of findings tables. J Clin Epidemiol 2011;64:383-94.

29. Balshem $H$, Helfand $M$, Schünemann $H J$, et al. GRADE guidelines: 3. rating the quality of evidence. J Clin Epidemiol 2011;64:401-6. 